\section{P29 Developing Nutrition Research Capacity at the Tanzania Food and Nutrition Centre through Collaborative Training and Technical Assistance}

Pamela Rothpletz-Puglia, EdD, RD, pr.puglia@rutgers.edu, Rutgers University, 65 Bergen Street, SSB 157, Newark, NJ 07107; Joyceline Kaganda, PhD, Tanzania Food and

Nutrition Centre

Objective: The Tanzania Food and Nutrition Centre (TFNC) formed a partnership with Rutgers School of Health Professions (SHP), Department of Nutritional Sciences (DNS) to build nutrition research capacity in systematic review and qualitative research skills.

Rationale: The TFNC organizes nutrition research in Tanzania and advises the Tanzanian government on key matters related to nutrition. Due to this responsibility, they sought continuing education in nutrition research methods from Rutgers faculty for capacity building.

Description: The TFNC director and a Rutgers faculty member participated in the in-person, 10 day WHO/Cochrane/Cornell Systematic Review Summer Institute for Systematic Reviews in Global Nutrition Policy Making to meet the systematic review learning need. Posttraining, a systematic review title and protocol were developed through remote communication with the author team. The qualitative research learning need was met with the provision of online education to TFNC staff. Rutgers faculty provided 3 months of education including modules on qualitative approaches, data collection, data analysis, and research protocol development.

Evaluation: The objectives for this research education training were met: A Rutgers faculty member and the TFNC director participated in systematic review training, and a protocol with TFNC and Rutgers authors was submitted to Cochrane for review. Twenty TFNC staff participated in qualitative research online education taught by Rutgers faculty, and the TFNC staff developed a qualitative nutrition research protocol.

Conclusions and Implications: It is feasible and productive to provide education and technical assistance through a combination of in-person and online training methods. The training resulted in sustainable research skills and in two research protocols that will be conducted collaboratively with TFNC staff and Rutgers faculty.

Funding: Rutgers Centers for Global Advancement and International Affairs (GAIA Centers) through an International Collaboration Research Grant, Rutgers SHP Department of Nutritional Sciences

\section{P30 Dietitians in the Kitchen Impact Cardiovascular Disease Prevention}

Nancy Tschiltz, MS, LDN, RD, Walter Reed Military

Medical Center; Joy Halsey, LDN, RD, CDE;

Arn Eliasson, MD, aheliasson@aol.com, Walter Reed
Military Medical Center, Cardiology Div, 8901 Wisconsin Avenue, Building 17, 2A, Bethesda, MD 20889; Mariam Kashani, DNP, CRNP, Walter Reed Military Medical Center, Bethesda, MD; Elaine Walizer, MSN, RN; Todd Villines, MD; Marina Vernalis, DO

Objective: To describe a successful dietetics intervention in an integrative cardiovascular health program.

Theory, Prior Research, Rationale: Amid the CVD epidemic, opportunities to learn basic cooking skills are decreasing while rates of eating out are continually increasing. Our research kitchen offers a unique opportunity to teach simple, Mediterranean-style, heart-healthy cooking techniques for use at home.

Description: The Integrative Cardiovascular Health Program (ICHP) follows evidence-based guidelines to measure CVD risk and contributory lifestyle behaviors. Subsequent interventions include a workshop, behavioral prescriptions, and coaching follow-up over 6 months, including appointments with a dietitian for diabetes education. The four-hour interactive workshop presents overviews on diet, exercise, stress management, and sleep. The program's capstone is a food preparation demonstration and Mediterranean-style meal in the research kitchen by a Culinary Institute of America-trained chef/Registered Dietitian. The goal is to teach simple recipes that taste good and empower attendees to plan, shop and prepare healthy meals at home.

Evaluation: After workshop participation, patients $(n=603$, mean age $57 \pm 12$ years, $44 \%$ men) expressed strong likelihood to try Mediterranean-style recipes (mean 3.8 of 4 points). Satisfaction was 99\% with the Mediterranean-style meal. Confidence was strong in 95\% of patients regarding where to shop for food. At program completion, 88 of 161 patients with prediabetes $(55 \%)$ normalized fasting glucose. Of 221 patients with hypercholesterolemia, 96 (43\%) normalized total cholesterol. Overweight patients $(n=491,81 \%)$ averaged $2.1 \mathrm{~kg}$ weight loss.

Conclusions and Implications: Interactive experiences in a research kitchen with dietitians teaching recipe planning, shopping, and meal preparation, enhance cardiovascular health outcomes.

Funding: The Henry M. Jackson Foundation for the Advancement of Military Medicine

\section{P31 Do Race and Insurance Matter? Exploring Differences in Response to an Educational Intervention on Cancer Prevention}

Alison Berg, PhD, RD, LD, alisoncberg@uga.edu, University of Georgia, 280 Dawson Hall, Athens, GA 30602; Swarn Chatterjee, PhD, University of Georgia

Objective: To explore relationships of demographics, insurance status, cancer screening history, and intention to implement cancer preventive behaviors (screening, nutrition, and physical activity) among participants of an 